\title{
Of mice (dogs) and men: getting to the heart of obesity-associated cardiac dysfunction
}

\author{
Christopher M. Haggerty ${ }^{1} \cdot$ Linyuan Jing $^{1} \cdot$ Brandon K. Fornwalt $^{1}$
}

Received: 9 October 2015 / Accepted: 14 October 2015 / Published online: 30 October 2015

(C) Springer-Verlag Berlin Heidelberg 2015

Keywords Animal models · Cardiac function ·

Cardiovascular disease $\cdot$ Heart $\cdot$ Insulin resistance $\cdot$ Magnetic resonance imaging $\cdot$ Obesity $\cdot$ Strain

\section{Abbreviation \\ LV Left-ventricular}

Obesity is a major public health problem, with over 600 million adults affected worldwide in 2014 [1]. The consequences of obesity are severe and include an elevated risk of early mortality [2]. Cardiovascular disease, including the development of heart failure, is a major contributor to the association between obesity and early mortality. Thus, significant research efforts have been directed at understanding the underlying mechanisms and potential treatment strategies for obesity-associated heart disease.

\section{Obesity-associated heart disease in humans}

Recent large cross-sectional studies have conclusively demonstrated a spectrum of left-ventricular (LV) remodelling that is associated with obesity. The remodelling is predominantly characterised by increased LV mass and mass-to-volume ratio (i.e. 'concentric' hypertrophy) [3, 4], which is generally considered to be a pathological form of remodelling. The exact

Brandon K. Fornwalt

bkf@gatech.edu

Institute for Advanced Application, Geisinger Clinic, $100 \mathrm{~N}$. Academy Ave., Danville, PA 17822, USA mechanism driving this remodelling is unclear, but elevated arterial blood pressure [5], hyperinsulinaemia [6] and fat/lipid infiltration [7] have all been causally implicated.

In addition to LV remodelling, many studies report impaired cardiac function in obese patients, including both diastolic and systolic dysfunction. Diastolic dysfunction, characterised by elevated atrial filling pressure and prolonged active relaxation, is presumably due to increased ventricular stiffness, and several studies have shown direct associations between diastolic dysfunction and BMI. Furthermore, recent evidence has demonstrated that diastolic dysfunction persists and worsens with a long-term ( 25 year) burden of obesity in adulthood [8].

With regard to systolic (i.e. contractile) function, many studies have shown that ejection fraction, the primary clinical marker of systolic function, is normal in the setting of obesity. However, advancements in non-invasive medical imaging make it possible to detect more subtle systolic dysfunction, which can occur despite normal ejection fraction. This subtle dysfunction includes impairments in myocardial strain, strain rate, torsion and synchrony of contraction (Fig. 1). Strain is a measure of the length change (shortening or lengthening) of the heart tissue along a particular axis (e.g. circumferential or longitudinal), while strain rate is the time rate of change in strain during contraction and relaxation. Torsion-commonly described with the analogy of wringing a wet towel-measures the spatial gradient of circumferential twist along the ventricular longitudinal axis. Synchrony quantifies the temporal uniformity of ventricular motion/contraction. These measures are collectively termed 'cardiac mechanics' and there is growing evidence of obesity-associated alterations in cardiac mechanics [5], with impaired longitudinal strain being the most consistent finding. Furthermore, data suggest that cardiac mechanics are better predictors of mortality than global measures such as ejection fraction [9]. Thus, impaired cardiac mechanics in patients 
Fig. 1 Normal heart contraction visualised with 'standard' and 'tagged' MRI. MR tagging uses spatial modulation of the magnetic field to superimpose a grid pattern on the signal produced by the tissue at a given instant in time (usually enddiastole). The deformation of the heart muscle deforms the grid, so observing the deformation of the grid elements - or tags - provides a direct measurement of myocardial motion. This quantitative deformation data can be used to compute cardiac mechanics, such as radial or circumferential strain and torsion/ twist
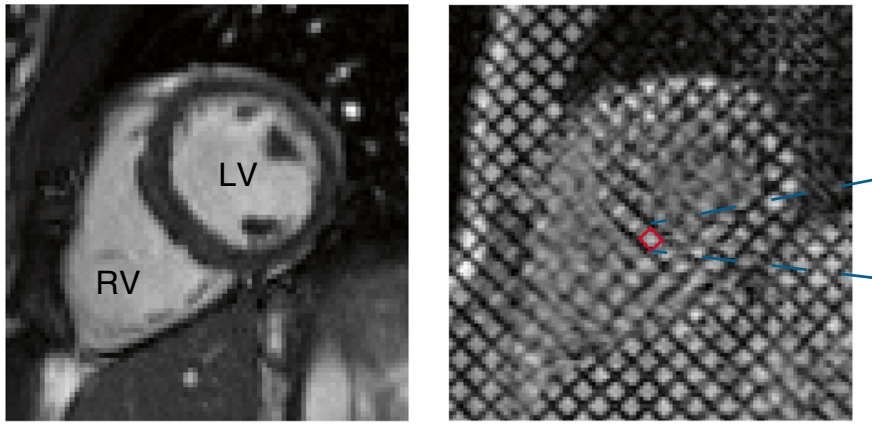

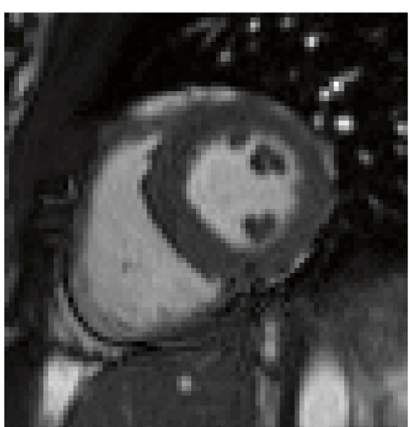

Standard MRI

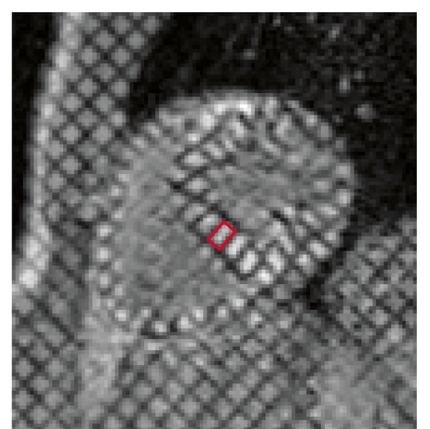

Tagged MRI

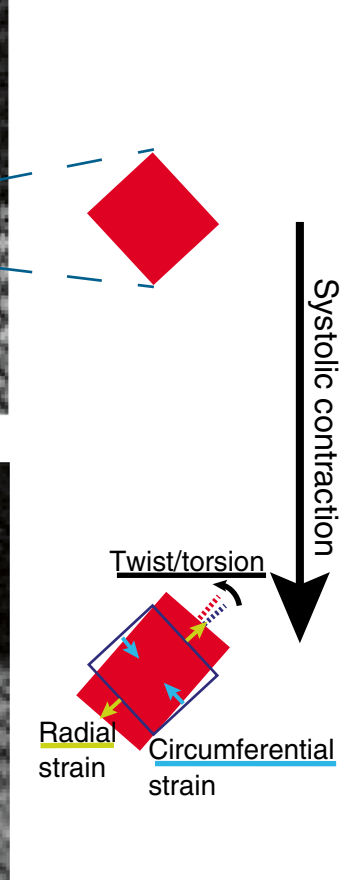

Deformation/strain with obesity may represent an early predictor of premature cardiovascular mortality.

Alarmingly, many of these pathological findings from obese adults have also been identified in obese children. Increased LV mass has been reported in obese children as young as 2 years of age [10], and studies in obese children and adolescents have reported both systolic and diastolic dysfunction [11-13]. The long-term implications of these findings in children remain unknown, although recent data from Franks et al showed that children in the highest BMI quartile had more than double the risk of premature death compared with children in the lowest BMI quartile [14]. These data offer compelling evidence that paediatric obesity is significantly detrimental to long-term health.

\section{Insights from animal models}

Many animal models, primarily rodents, have been studied with the intent of elucidating the mechanisms underlying obesity-associated cardiac remodelling and dysfunction. These models have generally been found to recapitulate common findings from human obesity, such as LV hypertrophy, systolic dysfunction [7, 15-17] and restricted coronary flow reserve [18]. Collectively, we have gained valuable insights from these studies, such as the mechanistic relationship between insulin resistance and cardiac remodelling/dysfunction $[6,15,19]$. Yet, in many ways, animal models have also demonstrated how profoundly complex the problem of obesity is, such as the finding that maternal obesity can lead to LV hypertrophy in offspring, independent of the weight or diet of the offspring [20].

In the current issue of Diabetologia, Broussard et al add to this collective experience with animal models of obesity and contribute interesting new insights [21]. Using a canine model of moderate obesity induced by lard-based high-fat feeding, the authors observed LV systolic dysfunction (i.e. impaired circumferential strain and torsion) via MRI, which manifested early (2-6 weeks) after diet initiation. Insulin resistance was also acutely elevated, consistent with their previous experience with this model. In fact, the authors demonstrated a moderate association between the acute insulin response and cardiac dysfunction, providing further evidence of their interrelationship. Finally, both insulin resistance and cardiac dysfunction were dependent on the source of dietary fat within the high-fat diet, as animals fed a salmon oil-based diet (i.e. polyunsaturated fatty acids) had normal cardiac function and insulin resistance despite similar weight gain between the two high-fat (lard vs salmon oil) groups.

The findings of this study are intriguing for several reasons. First, the authors had a sound experimental design that included concurrent measurements of arterial blood pressure and cardiac function via MRI. Elevated blood pressure is a frequent comorbidity of obesity that may confound assessment of cardiac function since strain, torsion, and ejection fraction are all load-sensitive measurements. Demonstrating impaired LV strain and torsion in the absence of elevated arterial blood pressures confirms that obesity-related cardiac dysfunction may occur independently of hypertension. Similarly, no cardiac structural remodelling (increased myocardial 
mass/chamber volume or myocardial steatosis) was observed in the lard group, indicating that cardiac dysfunction is not strictly a consequence of remodelling. These findings are somewhat limited by the fact that no measures of longitudinal strain were performed, and impaired longitudinal strain is the most consistent systolic function abnormality demonstrated in human obesity. Thus, the independence of impaired longitudinal strain from hypertension and LV remodelling can only be assumed.

Another intriguing implication of the study by Broussard et al is to what extent obesity contributed to the observed impairments in cardiac function. The acuteness of the responses ( 2 weeks) coupled with the fact that the dependence on the dietary fat source was greater than that on the very moderate amount of body weight gained suggests that 'obesity' in this model is just another confounding variable. Instead, perhaps direct diet-induced effects such as inflammation and/or insulin resistance $[19,22]$ may be the primary driving forces behind the pathogenesis of abnormal cardiac mechanics, at least at such an acute stage. Additional research is obviously needed to evaluate such a hypothesis.

\section{Reconciling human and animal disease}

It is instinctive, and easy, given the direct compatibility of MRI measurements between animals and humans, to directly extrapolate from studies such as that of Broussard et al [21] and others $[16,17]$ to human disease, but such an exercise is fraught with challenges. For example, while reduced cardiac mechanics in animals are generally consistent with findings in human studies, the details of reduced circumferential strain and torsion are subject to debate, as some human studies have found no change [12] or even increases [13] in those specific measures. The significance of these discrepancies cannot be determined based on current knowledge. In addition, it is difficult to predict the extent to which the processes governing an acute response to a high-fat diet in animals are similar to and representative of the processes governing a chronic response to obesity in humans. In fact, mice fed a high-fat diet may have an acute increase in myocardial strain and torsion after 4-10 weeks [16], suggesting that there may in fact be inter-species differences in these responses. Furthermore, while cardiac dysfunction can occur independently of cardiac remodelling or arterial hypertension, it cannot be stated that cardiac dysfunction in humans is in fact free of such interactions, because these factors are often present and will affect function. Hence, such detailed answers to disease pathogenesis must ultimately come from human studies.

Rather than attempting such direct comparisons, a more synergistic interpretation may be helpful. Two theories clearly extend from the work by Broussard et al [21] in concert with other recent human and animal investigations: (1) using appropriately sensitive measurement techniques, such as motion-sensitive MRI, cardiac mechanics of the LV are impaired in the setting of obesity; and (2) insulin resistance is a critical element-at least as one of several potentially causative or intermediary factors - in the linkage between obesity and heart disease. The immediate knowledge gaps that extend from these theories are then fairly straightforward: (1) what is the long-term prognostic significance of reduced cardiac mechanics in obese patients; and (2) can therapeutically targeting insulin signalling pathways positively impact the adverse effects of obesity on the heart? These are the questions that must be tackled as we continue to struggle with the impact of the obesity epidemic.

Funding This work was supported by the National Heart, Lung, and Blood Institute of the National Institutes of Health under Award Number F32HL123215, the National Institute of General Medical Science of the National Institutes of Health under Award Number P20GM103257 and by the American Heart Association under Award Number 14POST20310025. The content is solely the responsibility of the authors and does not necessarily represent the official views of the funding sources.

Duality of interest The authors declare that there is no duality of interest associated with this manuscript.

Contribution statement All authors were responsible for drafting the article and revising it critically for important intellectual content. All authors approved the version to be published.

\section{References}

1. World Health Organization (2015) Obesity and overweight. Fact sheet no. 311. Available from http://www.who.int/mediacentre/ factsheets/fs311/en/, accessed 28 September 2015

2. Flegal KM, Graubard BI, Williamson DF, Gail MH (2007) Causespecific excess deaths associated with underweight, overweight, and obesity. JAMA 298:2028-2037

3. Avelar E, Cloward TV, Walker JM et al (2007) Left ventricular hypertrophy in severe obesity: interactions among blood pressure, nocturnal hypoxemia, and body mass. Hypertension 49:34-39

4. Turkbey EB, McClelland RL, Kronmal RA et al (2010) The impact of obesity on the left ventricle. J Am Coll Cardiol Img 3:266-274

5. Aurigemma GP, de Simone G, Fitzgibbons TP (2013) Cardiac remodeling in obesity. Circ Cardiovasc Imaging 6:142-152

6. Bertrand L, Horman S, Beauloye C, Vanoverschelde JL (2008) Insulin signalling in the heart. Cardiovasc Res 79:238-248

7. Abel ED, Litwin S, Sweeney G (2008) Cardiac remodeling in obesity. Physiol Rev 88:389-419

8. Kishi S, Armstrong AC, Gidding SS et al (2014) Association of obesity in early adulthood and middle age with incipient left ventricular dysfunction and structural remodeling. JACC Heart Fail 2: 500-508

9. Stanton T, Leano R, Marwick TH (2009) Prediction of all-cause mortality from global longitudinal speckle strain: comparison with ejection fraction and wall motion scoring. Circ Cardiovasc Imaging 2:356-364

10. de Jonge LL, van Osch-Gevers L, Willemsen SP et al (2011) Growth, obesity, and cardiac structures in early childhood: the Generation R Study. Hypertension 57:934-940 
11. Cote AT, Harris KC, Panagiotopoulos C, Sandor GGS, Devlin AM (2013) Childhood obesity and cardiovascular dysfunction. J Am Coll Cardiol 62:1309-1319

12. Koopman LP, McCrindle BW, Slorach C et al (2012) Interaction between myocardial and vascular changes in obese children: a pilot study. J Am Soc Echocardiogr 25:401-410

13. Saltijeral A, Isla LP, Perez-Rodriguez O et al (2011) Early myocardial deformation changes associated to isolated obesity: a study based on 3D-wall motion tracking analysis. Obesity (Silver Spring) 19:2268-2273

14. Franks PW, Hanson RL, Knowler WC, Sievers ML, Bennett PH, Looker HC (2010) Childhood obesity, other cardiovascular risk factors, and premature death. N Engl J Med 362:485-493

15. Noyan-Ashraf MH, Shikatani EA, Schuiki I et al (2013) A glucagon-like peptide-1 analog reverses the molecular pathology and cardiac dysfunction of a mouse model of obesity. Circulation 127:74-85

16. Haggerty CM, Mattingly AC, Kramer SP et al (2015) Left ventricular mechanical dysfunction in diet-induced obese mice is exacerbated during inotropic stress: a cine DENSE cardiovascular magnetic resonance study. J Cardiovasc Magn Reson 17:75

17. Kramer SP, Powell DK, Haggerty CM et al (2013) Obesity reduces left ventricular strains, torsion, and synchrony in mouse models: a cine displacement encoding with stimulated echoes (DENSE) cardiovascular magnetic resonance study. J Cardiovasc Magn Reson 15:109

18. Naresh NK, Chen X, Roy RJ, Antkowiak PF, Annex BH, Epstein FH (2015) Accelerated dual-contrast first-pass perfusion MRI of the mouse heart: development and application to diet-induced obese mice. Magn Reson Med 73:1237-1245

19. Fu Q, Xu B, Liu Y et al (2014) Insulin inhibits cardiac contractility by inducing a $\mathrm{G}_{\mathrm{i}}$-biased $\beta_{2}$-adrenergic signaling in hearts. Diabetes 63:2676-2689

20. Fernandez-Twinn DS, Blackmore HL, Siggens L et al (2012) The programming of cardiac hypertrophy in the offspring by maternal obesity is associated with hyperinsulinemia, AKT, ERK, and mTOR activation. Endocrinology 153:5961-5971

21. Broussard JL, Nelson MD, Kolka CM et al (2015) Rapid development of cardiac dysfunction in a canine model of insulin resistance and moderate obesity. Diabetologia. doi:10.1007/s00125-0153767-5

22. Chess DJ, Stanley WC (2008) Role of diet and fuel overabundance in the development and progression of heart failure. Cardiovasc Res 79:269-278 